\title{
Optimal management of steroid-dependent ulcerative colitis
}

This article was published in the following Dove Press journal:

Clinical and Experimental Gastroenterology

12 November 2015

Number of times this article has been viewed

\author{
Hafiz M Waqas Khan' \\ Faisal Mehmood' \\ Nabeel Khan ${ }^{2}$ \\ 'Department of Medicine, King \\ Edward Medical University, \\ Lahore, Pakistan; ${ }^{2}$ Section of \\ Gastroenterology, University of \\ Pennsylvania Perelman School of \\ Medicine, Philadelphia VA Medical \\ Center, Philadelphia, PA, USA
}

\begin{abstract}
Ulcerative colitis (UC) is a chronic inflammatory condition that is variable in both extent and severity of disease as well as response to therapy. Corticosteroids (CSs) were the first drugs used in the management of UC and are still used for induction of remission. However, because of their extensive side-effect profile, they are not utilized for maintenance of remission. In view of this, CS-free remission has become an important end point while evaluating therapeutic agents used in the management of UC. This review highlights the results of various studies conducted to evaluate the efficacy of different medications to attain CS-free remission in the setting of active UC. The drugs reviewed include established agents such as thiopurines, methotrexate, infliximab, adalimumab, vedolizumab, golimumab, and newer experimental agents, and if all else fails, colectomy will be performed. The efficacy of these drugs is evaluated individually. Our aim is to provide a synopsis of the work done in this field to date.
\end{abstract}

Keywords: ulcerative colitis, steroid dependent, thiopurines, MTX, adalimumab, infliximab

\section{Ulcerative colitis}

Ulcerative colitis (UC) is an inflammatory disorder of the colon which follows a relapsing and remittent course. The primary area of involvement of this disease is rectum, but it can also spread to other parts of the colon in a proximal fashion. ${ }^{1}$ The disease shows a variable behavior in terms of its extent, severity, and clinical course. ${ }^{1}$ The exact etiology of UC is still unknown, but some environmental factors have been considered to play a role in its etiology.,3

\section{Epidemiology of UC}

Approximately, 1.3 million people suffer from inflammatory bowel disease (IBD) in the US. ${ }^{4-7}$ Different studies have reported the incidence and prevalence rates per 100,000 persons of the US population, which are $2.2-14.3$ and $37-246$, respectively. ${ }^{4-7}$

Molodecky et $\mathrm{al}^{5}$ reported in their study that incidence and prevalence of UC were highest in Canada (19.2 per 100,000), Northern Europe (24.3 per 100,000), and Australia (17.4 per 100,000). Similarly, prevalence was highest in Europe (505 per 100,000) and Canada (248 per 100,000). They reviewed 108 epidemiological studies conducted through a period of 1930-2010 and found that most studies (51.1\%) reported the highest incidence in third decade of life.

\section{Corticosteroids and UC}

Corticosteroids (CSs) were first studied by Truelove and Witts ${ }^{8}$ who conducted a trial in UC patients with active disease. They treated the patients with $100 \mathrm{mg}$ cortisone 
daily and then tapered it in next 6 weeks and found it an effective treatment. Nearly 60 years since discovery, CS treatment remains the first line and intervention of choice in UC patients. ${ }^{9}$ Because of their high efficacy, CS are often used in the initial treatment of moderate-to-severe UC. ${ }^{10}$ A dose of $40 \mathrm{mg}$ of prednisone daily has been found to be effective in induction of remission in patients with moderateto-severe UC. ${ }^{10} \mathrm{CS}$-responsive UC is defined as the disease which responds to high doses (HDs) of CS within 1 month or 1 week of oral or intravenous CS therapy, respectively. ${ }^{11-13}$ At molecular level, CS bind with the intracellular glucocorticoid receptor and exert their anti-inflammatory and immunesuppressant actions by inhibiting the T-cell activation and proinflammatory cytokines which play an important role in the pathogenesis of UC. ${ }^{13}$

However, irrespective of efficacy, CS are associated with short-term and long-term side effects. ${ }^{10}$ Among the shortterm side effects, highest incidence has been reported of ecchymosis, infections, acne, and moon face in $17 \%, 27 \%$, $30 \%$, and $47 \%$ of the patients, respectively. ${ }^{10}$ Commonly observed long-term effects are steroid-associated osteoporosis, glaucoma, cataracts, and new-onset diabetes mellitus and hypertension. ${ }^{10}$ Limited data are available on the reversibility of side effects of CS based on decreasing the dosage and alternate day dosage, but no definite results have been reached yet as to whether short-term effects are reversible and treatable. ${ }^{10}$ Thus, in view of this extensive side-effect profile, $\mathrm{CS}$ are not considered as agents for long-term management of UC, and CS-free remission has become an important focus of UC trials.

\section{Steroid-dependent (SD)-UC}

$\mathrm{SD}-\mathrm{UC}$ is defined as the disease that initially responds to CS but could not maintain control of symptoms without $\mathrm{CS}$ and needs low doses (LDs) of CS to remain symptom free. ${ }^{11-13}$

Faubion et $\mathrm{al}^{11}$ identified 185 patients with UC from 1970 to 1993 who were treated with systemic CS in Olmsted County, Minnesota, and determined the outcome of patients at 1 year after treatment with first CS therapy. They found that $34 \%$ of UC patients required CS and found that immediate outcomes were complete remission, partial remission, and no response in $54 \%, 30 \%$, and $16 \%$, respectively, while longterm outcomes were prolonged response, CS dependence, and surgery in $49 \%, 22 \%$, and $29 \%$, respectively.

Similarly, Ho et al ${ }^{14}$ conducted a trial to determine the response of patients after treatment with first CS therapy. They performed multivariate analysis on $136 \mathrm{UC}$ patients identified in 5-year inception cohort and found that $63 \%$ of UC patients required CS therapy. They also reported that at 30 days, complete response, partial response, and no response were observed in $51 \%, 31 \%$, and $18 \%$ of patients, respectively, and prolonged response, CS dependence, and surgery were the outcomes in $55 \%, 17 \%$, and $21 \%$ of patients, respectively, at 1 year.

Another study was performed by Khan et al ${ }^{15}$ to assess the prevalence of CS use and long-term outcomes after the index CS exposure. One thousand and thirty-eight patients were identified using the database from the health care system of Veterans Affairs. They found that the prevalence of CS use was 45\%. They included 464 CS users and followed them over a median period of 3.4 years and reported that $65 \%$ required CS reintroduction, $38 \%$ were classified as CS dependent, and $11 \%$ were classified as CS refractory mostly within 2 years after the index CS course. A separate study by Khan et $\mathrm{al}^{16}$ also evaluated the role of early CS treatment in the development of CS dependency. They followed 23\% of the patients with early CS treatment over a median time of 4.7 years and reported that the patients who received early CS treatment had higher percentages of CS dependency than who did not receive early treatment $(P<0.001)$.

In view of the side effects of $C S$, different drugs are used for the management of SD-UC. These drugs are summarized in the following section.

\section{Overview of drugs used in SD-UC Thiopurines}

Azathioprine (AZA) is analogous to purine nucleotides and affects their biosynthesis by mechanism of competitive inhibition. ${ }^{17}$ Thiopurines (TPs) have been considered the reference treatment in patients with SD moderateto-severe IBD for many years. ${ }^{18}$ TPs can cause a number of side effects that could be dose related, such as leukopenia and hepatitis, and non-dose related, such as fever, rash, nausea, and pancreatitis. ${ }^{17}$ Several different trials assessed the role of AZA in SD-UC patients.

Kirk and Lennard-Jones ${ }^{19}$ were the first to conduct a trial of AZA among 44 patients with active UC taking prednisolone at baseline and found that CS dose reduction was observed to be significantly higher in AZA-treated patients than placebo after 6-month follow-up $(P<0.001)$. Similarly, Adler and Korelitz ${ }^{20}$ conducted a study on 81 CS-dependent patients with resistant disease who were treated with 6-mercaptopurine (6-MP) for minimum of 4 months over 18 years (1970-1988) and reported that CS discontinuation and reduction were achieved in $48 \%$ and 
$13 \%$, respectively. Another trial performed by Sood et $\mathrm{al}^{21}$ on 83 patients with severe UC found that AZA when used with CS decreased the number of relapses with no effect on remission rates. Maté-Jiménez et $\mathrm{al}^{22}$ compared the efficacy 6-MP, methotrexate (MTX), and 5-ASA in achieving and maintaining the $\mathrm{CS}$-free remission in $34 \mathrm{SD}-\mathrm{UC}$ patients with baseline CS and found that 6-MP was superior than 5-ASA in achieving remission ( $78 \%$ vs $25 \%$, respectively, $P<0.05$ ) and superior than 5-ASA and MTX in maintaining remission $(63 \%, 14.3 \%$, and $0 \%$, respectively, $P<0.0015)$.

A large 30 -year review was performed by Fraser et al ${ }^{17}$ to assess the role of AZA in the management of IBD. Clinical notes of 2,205 patients who attended the hospital from 1968 to 1999 were reviewed. They found that a total of 622 patients were treated with AZA and 346 had UC. They reported that CS-free remission was observed in 58\% in overall AZA-treated patients and $87 \%$ among those who completed 6 months treatment.

Ardizzone et $\mathrm{al}^{23}$ compared the efficacy of AZA and 5-ASA for induction of remission in patients with SD-UC. Seventytwo patients received systemic prednisolone and followed up for 6 months. They found AZA to be more superior than 5-ASA in achieving remission and discontinuation of CS in the intention-to-treat ( $53 \%$ vs $21 \%, 95 \%$ confidence interval [CI] $1.57-14.5$ ) and in the protocol ( $58 \%$ vs $21 \%, 95 \%$ CI $1.59-18.1$ ) analysis. Khan et $\mathrm{a}^{24}$ performed a literature review and analyzed two AZA randomized controlled trials in 130 active UC patients and three trials in 127 quiescent UC patients. They reported that AZA was found statistically significant in prevention of relapses (RR [relative risk] 0.60, 95\% CI 0.37-0.95); however, results did not achieve statistical significance in induction of remission (RR 0.85, 95\% CI 0.71-1.01).

Toronto UC Consensus Group ${ }^{25}$ recently performed a thorough search on literature and identified studies on the management of UC. They recommended against the use of TPs monotherapy in induction of CS-free remission but in selected patients with UC who have achieved symptomatic remission on oral CS. They suggest TPs-monotherapy as an option to maintain CS-free remission.

Recently, Panaccione et $\mathrm{al}^{26}$ performed the SUCCESS trial and looked at the efficacy of infliximab (IFX) and AZA alone and in combination to achieve CS-free remission, and found that at week 16, 23.7\% of TPs-treated patients were in CS-free remission at week 16.

\section{Methotrexate}

MTX is an immunosuppressant (IS) used in the treatment of UC and decreases inflammation by some unknown mechanisms. Mechanisms generally considered possible for MTX actions are cellular inhibition, inhibition of inflammatory mediators, clonal deletion of activated peripheral T-cells by CD95-independent pathway, and apoptosis. ${ }^{27-29}$ MTX can cause stomatitis, nausea, and diarrhea in $5 \%$ of the patients. ${ }^{29}$ Few studies have reported Epstein Barr Virus-related lymphoproliferative disorders and rarely hepatotoxicity. ${ }^{29}$ Many trials have been conducted to evaluate the role of MTX in achieving clinical and CS-free remission.

Kozarek et $\mathrm{al}^{30}$ were the first to perform a nonrandomized trial to assess the efficacy of MTX in seven patients with chronic refractory UC by treating them with MTX for 12 weeks. They reported that $71.4 \%$ of patients with $\mathrm{UC}$ had an objective response $(P=0.007)$ and were able to significantly reduce their CS dose after treatment with $\operatorname{MTX}(P=0.01)$.

Another study conducted by Oren et $\mathrm{al}^{31}$ did not find MTX as a useful drug and found no significant difference in MTXand placebo-treated patients. Sixty-seven UC patients were included in the study on the basis of their use of CS and IS in the preceding year and treated with MTX for 9 months. The authors found that results did not achieve statistical significance and observed no difference between MTX and placebo in entering first remission $(46.7 \%$ vs $48.6 \%$, respectively), time to achieve first remission (4.1 \pm 1.9 months vs $3.4 \pm 1.7$ months, respectively), and failure after first remission (64.3\% vs $44.4 \%)$.

Many small studies established the role of MTX in achieving CS-free remission. Paoluzi et $\mathrm{al}^{32}$ conducted a trial in ten patients with SD-UC or steroid-refractory UC who were AZA intolerant/resistant and treated them with MTX for 6 months. They found that $100 \%$ of the patients discontinued CS in short-term results, and none of the patients with complete response relapsed in the long-term follow-up, while $50 \%$ with partial response relapsed. Similarly, Cummings et $\mathrm{al}^{33}$ investigated the efficacy of MTX in achieving CS-free remission by reducing their CS dose calculated as the response. In the 42 patients with UC (31 AZA intolerant, eleven AZA nonresponders), they reported the efficacy as good in $54 \%$ and partial in $18 \%$ of patients. Overall, CSfree remission was achieved and sustained for $>6$ months in $42 \%$ and $38 \%$ of the patients, respectively. Wahed et $\mathrm{al}^{34}$ conducted a similar trial in 32 UC patients (23 TPs intolerant, nine TPs nonresponders) who were taking CS at baseline (55.65\% and $60.8 \%$, respectively) and found that CS withdrawal was possible in $36.8 \%$ of patients. Mañosa et al ${ }^{35}$ analyzed data of $40 \mathrm{UC}$ patients from different hospitals who were treated with MTX, and found that out of $85 \%$ of the 
patients who were taking CS at baseline, 60\%, 48\%, and 35\% of the patients maintained CS-free remission at 6,12 , and 24 months, respectively. Similarly, Baron et $\mathrm{al}^{36}$ conducted a study in eight patients with SD-UC and treated them with oral MTX and observed statistically significant reduction in daily prednisone dose from 26.3 to $12.7 \mathrm{mg}$ per day with MTX use $(P<0.001)$.

A recent cohort study was conducted by Khan et $\mathrm{al}^{37} \mathrm{using}$ the Veterans Affairs database for the period of 2001-2011. They identified 91 SD-UC patients who were using MTX and CS. Patients were followed for 15 months after their initiation of MTX and were studied for their response to discontinue CS. After 15-month follow-up, discontinuation of CS was observed in $30 \%$ and $37 \%$ of the patients who used parenteral and oral MTX, respectively. A review of trials has been performed recently by $\mathrm{Chande}^{38}$ to evaluate the role of MTX in achieving CS-free remission in UC from inception to June 26, 2014, and found statistically no significant difference between MTX and placebo (47\% vs 49\%, respectively) and MTX, 6-MP, and 5-ASA (58\%, 79\%, and $25 \%$, respectively).

Recently, Carbonnel ${ }^{39}$ conducted a trial on the efficacy of MTX in patients with SD-UC and assessed its role in achieving clinical as well as CS-free remission without further need of other therapies such as IS, antitumor necrosis factors (anti-TNFs), or colectomy. They included 111 patients in 26 European centers with $25 \mathrm{mg}$ per day median dose of prednisone at inclusion, and found that MTX did not achieve statistical significance in achieving primary end point, but MTX (42\%) achieved significantly higher rates of CS-free clinical remission without the need for other therapies as compared to placebo $(23.5 \%)$ in SD-UC patients at 16 weeks $(P=0.04)$.

\section{Infliximab}

IFX is a monoclonal antibody which has high affinity and specificity for TNF- $\alpha$ and has the ability of binding to its soluble as well as the membrane-bound forms and prevents its biological functions involved in immune responses in UC. ${ }^{40,41}$ TNF- $\alpha$ is a proinflammatory cytokine produced by macrophages and $\mathrm{T}$ lymphocytes and increases cell migration by expressing the adhesion molecules on endothelial cells. IFX prevents its functions and decreases the inflammation in UC. ${ }^{41}$ It is the first biological agent approved by the US Food and Drug Administration (FDA) for the management of UC as induction and maintenance therapy and is effective in induction and maintenance of UC. ${ }^{42}$ Many adverse events (AEs) have been reported with its use with the greatest incidence of infusion reactions due to antibodies, serum sickness, increased risk of opportunistic infections, and reactivation of latent tuberculosis. ${ }^{41}$ Different controlled trials have studied the role of IFX in the treatment of UC and its efficacy in CS-free remission. ${ }^{42-49}$

ACT 1 and ACT 2 were two large trials that investigated the efficacy of IFX in 364 UC patients with moderate-tosevere disease. These patients either failed CS treatment or were intolerant to them at baseline of the study. ${ }^{42}$ Rutgeerts et $\mathrm{al}^{42}$ reported that $61 \%$ and $51.1 \%$ of patients had baseline CS use in ACT 1 and ACT 2 trials, respectively. They reported that at week 30 , patients who discontinued the use of CS in ACT 1 and ACT 2 were $21.7 \%$ and $22.8 \%$, respectively, in the IFX subgroups, and at week $54,21 \%$ of patients discontinued the use of CS in ACT 1. Results showed higher percentages of patients with CS-free clinical remission with IFX than placebo.

Probert et $\mathrm{al}^{45}$ conducted a trial to assess the efficacy of IFX in patients with SD-UC as compared to placebo. They treated the patients with two doses of IFX and assessed remission using Ulcerative Colitis Symptom Score and Baron score at 6 weeks. They found that most patients were able to reduce their dose of CS in different subgroups of the study. They reported that patients in IFX-treated groups were able to significantly reduce their CS dose with a mean reduction of $19 \mathrm{mg}$ as compared with the placebo group (14 mg, $P=0.037$ ).

Many small studies supported the efficacy of IFX in SD-UC. Bermejo et $\mathrm{al}^{50}$ presented seven cases of UC treated with IFX. Out of these seven patients, six had SD-UC. They reported that five out of six patients $(83.3 \%)$ were successfully weaned off these drugs. Another study was conducted by Kountouras et $\mathrm{al}^{51}$ to assess the role of IFX in eight patients with SD-UC. They treated the patients with IFX $5 \mathrm{mg} / \mathrm{kg}$ and followed them for 3 years and reported discontinuation of CS in all eight patients (100\%). Barreiro-de Acosta et $\mathrm{al}^{52}$ performed a study to assess the efficacy of IFX in SD-UC. They treated 17 SD (European Crohn's and Colitis Organization criteria) patients with IFX, and found that at week 104, 70\% of patients sustained CS-free clinical remission $(P<0.01)$. Another study was conducted by Willert and Lawrance ${ }^{53}$ in which they enrolled 15 patients who had SD-UC and steroid-refractory UC to study the use of IFX. Patients were also using concomitant AZA/6-MP and ASA. They reported that response to IFX was observed in $86.7 \%$ of the patients and $40 \%$ of these patients discontinued CS at week 8. Mocciaro et $\mathrm{al}^{54}$ studied efficacy of IFX in moderate-to-severe SD-UC by treating 13 patients 
with $5 \mathrm{mg} / \mathrm{kg}$ of IFX and observed that $54 \%$ and $61.5 \%$ of patients achieved clinical remission and discontinued CS, respectively.

Two larger studies ${ }^{26,55,56}$ were recently conducted by Armuzzi et al and Panaccione et al to study the role of IFX and AZA in achieving CS-free remission in patients with SD-UC. Armuzzi et al ${ }^{55}$ conducted a trial in 126 SD-UC patients and treated them with IFX. The end points were CS-free remission and mucosal healing at 6 months and 12 months. CS-free remission was observed in $53 \%$ and $47 \%$ of patients at 6 and 12 months, respectively. Thirty-two percent achieved CS-free remission and mucosal healing at 12 months. In another study, ${ }^{56}$ they further followed the patients for 42 months to see long-term outcomes, and reported that out of 96 patients who responded to IFX, CS-free clinical response was sustained by $65 \%$ and $42 \%$ of patients at 42 months and last follow-up, respectively. They also reported that higher percentages of patients were observed to maintain CS-free clinical response when treated with combined therapy of IFX and AZA rather than alone. Recently, Panaccione et $\mathrm{al}^{26}$ also conducted a similar trial to assess the efficacy of IFX and AZA alone and in combination. They reported that $39.7 \%$ of patients achieved CS-free remission in the combination group, a significantly greater percentage as compared to groups treated alone with IFX and AZA at week 16 (22.1\% and $23.7 \%, P=0.017$ and 0.037 , respectively).

\section{Adalimumab}

This monoclonal antibody to TNF- $\alpha$ is fully humanized and is approved by the FDA for the treatment of moderate-tosevere UC since 2012. It has the same mechanism of action as described earlier for IFX. Observed side effects were injection site reactions, allergic reactions, and opportunistic infections. ${ }^{57}$ Different studies have been conducted to assess the role of adalimumab (ADA) in UC in achieving clinical as well as CS-free remission. ${ }^{57-60}$

ULTRA 1 and ULTRA 2 were two controlled trials that investigated the role of ADA in induction of remission and maintenance of remission in patients with UC. ${ }^{59,61}$ Sandborn et $\mathrm{al}^{59}$ conducted the ULTRA 2 trial to assess the efficacy of ADA in UC patients with moderate-to-severe disease. Four hundred and ninety-four patients were included who had also received CS and IS therapies at baseline. They found that at week $52,13.3 \%$ of patients discontinued CS use and achieved CS-free remission $(P=0.03$ ), while $10 \%$ discontinued CS use and achieved sustained remission at both weeks 32 and $52(P=0.002)$.

Sandborn et $\mathrm{al}^{62}$ also assessed the rates of CS-free remission and $\mathrm{CS}$ discontinuation in subgroups of patients who achieved clinical response at 8 weeks in ULTRA 2 trial and observed that among the responders using $\mathrm{CS}$ at baseline, $21.1 \%$ achieved CS-free remission and $37.8 \%$ were CS free at week 52. Colombel et $\mathrm{al}^{63}$ collected data of patients who used CS at baseline from ULTRA trials and identified those patients who received ADA for 4 years and assessed CS-free remission in them. Out of 600 patients who received ADA, 199 patients received ADA for 4 years. They concluded that $59.2 \%$ of patients discontinued CS at week 208 .

Another study was conducted by Acosta ${ }^{64}$ which described the role of ADA in SD-UC patients. They conducted a multicenter trial and included 37 SD patients $>18$ years of age. Patients were treated with ADA for induction and followed over a mean period of 25.9 months. The author observed that remission was achieved in $35 \%$ of the patients after induction and after 12 months, and $40 \%$ of patients achieved CS-free remission. Tursi et $\mathrm{al}^{65}$ performed a trial on effectiveness of ADA in UC patients and reported CS-sparing effects of ADA. They enrolled 15 patients treated with ADA and reported that ten patients achieved CS-free remission and discontinued their steroids at week 54 (67.3\%).

A very recent retrospective multicenter study has been conducted by Taxonera et $\mathrm{al}^{66}$ to assess the role of ADA dose escalation in $200 \mathrm{UC}$ patients who failed to respond to ADA and required dosage escalation. They found that weekly increase in the dosage was required in $38 \%$ of the patients. They also reported that short-term clinical response and clinical remission were observed in $47 \%$ and $20 \%$ of the patients, respectively $(P<0.001)$. They reported that patients who had short-term response after increasing the dose of ADA had a decreased percentage of ADA failure and colectomy $(56 \%$, HR [hazard ratio] $0.60,95 \%$ CI $0.12-0.80, P<0.01$ and $21 \%$, HR 0.53 , 95\% CI 0.03-0.69, $P<0.007$, respectively).

\section{Vedolizumab}

Vedolizumab is a monoclonal antibody against $\alpha 4 \beta 7$ integrin molecule. ${ }^{67}$ The $\alpha 4 \beta 7$ integrin is expressed on $\mathrm{T}$ lymphocytes and plays an important role in their recruitment to the intestines as well as their function in UC. ${ }^{67}$ It binds with the integrin molecule and inhibits the T-cell recruitment suppressing the inflammatory functions of T-cells in UC. ${ }^{67}$ Intravenous vedolizumab is approved in several countries including the USA and European Union for the management of active UC with moderate-to-severe disease and is the first integrin antagonist to be approved for the treatment of IBD. ${ }^{68}$ No significant difference was observed in the development of adverse effects in patients taking vedolizumab or placebo. Headache, abdominal pain, arthralgia, dizziness, rash, and 
infusion reaction with angioedema were observed in all the groups ${ }^{67}$ Different studies that investigated the efficacy of vedolizumab are given in the following section.

The GEMINI 1 study was a large randomized, international, multicenter trial conducted by Feagan et a ${ }^{69}$ that included separate induction and maintenance portions. Patients between 18 and 80 years of age and who had active $\mathrm{UC}$ and failed one or more previous treatments (CS, IS, anti-TNFs) were enrolled between 2008 and 2012. The study included $36.7 \%$ and $16.5 \%$ of the patients taking CS only and CS and IS together at baseline, respectively, and found that in the maintenance phase, both 4-week and 8-week vedolizumab arms achieved superior CS-free remission rates $(45.2 \%$ and $31.4 \%$ vs $13.9 \%$ in the placebo group, $P<0.001$ and $P=0.01$, respectively).

Parikh et $\mathrm{al}^{70}$ conducted a study on long-term effects of vedolizumab in UC. They randomized patients with UC to vedolizumab and followed up them every 8 weeks. They reported that at baseline, 19 patients were receiving oral CS, primarily prednisone. Analysis showed that 12 of these patients (63\%) were able to discontinue CS completely while on study and remained CS free at their last assessment. An additional three patients were able to decrease their dose by more than $50 \%$. Overall, 15 patients $(79 \%)$ were able to substantially reduce or discontinue CS use.

\section{Golimumab}

This monoclonal antibody is also humanized which binds to both transmembrane and soluble forms of TNF- $\alpha$, inhibits its functions similarly in the way of IFX and ADA, and received the FDA approval in May 2013 for inducing and maintaining CS-free remission in moderate-to-severe UC. Because of its subcutaneous route of administration, patients can self-administer it at home. ${ }^{71}$ Most frequently observed side effects with the use of golimumab are headache, nasopharyngitis, abdominal pain, opportunistic infections, and reaction of tuberculosis. ${ }^{72,73}$ Studies that investigated the role of golimumab in induction and maintenance of remission are given in the following section.

PURSUIT-SC and PERSUIT-M were two large trials that investigated the role of golimumab in the induction and maintenance of remission, respectively, in patients with UC..$^{72,73}$ Sandborn et $\mathrm{al}^{73}$ reported in PURSUIT-M study that $\sim 54 \%$ of patients were taking CS at baseline. The percentages of patients in CS-free remission at week 54 were $23.2 \%, 28.2 \%$, and $18.4 \%$ in the $100,50 \mathrm{mg}$, and placebo groups, respectively. The number needed to treat for patients achieving CS-free remission at week 54 was 21 and 10 for the $100 \mathrm{mg}$ and
$50 \mathrm{mg}$ golimumab groups, respectively. They also reported in PURSUIT-M study that among golimumab-induction responders, $51.6 \%$ and $54.3 \%$ were taking CS at baseline in 50 and $100 \mathrm{mg}$ groups, respectively. Of these, more number of patients in the $100 \mathrm{mg}$ group (38.5\%) as well as in the $50 \mathrm{mg}$ group (38.5\%; nominal $P=0.026$ ) maintained CS-free clinical response through week 54 than placebo group (20.7\%).

Limited data are available regarding the efficacy of golimumab in SD-UC. However, few trials are ongoing about the role of golimumab in SD-UC but are not completed yet.

\section{Newer therapies \\ Sphingosine- I-phosphate receptor modulator (RPCI063)}

RPC1063 is an oral therapy that acts as a selective receptor modulator of sphingosine-1-phosphate receptors and modulates the distribution of T-cell lymphocytes exerting its anti-inflammatory actions. ${ }^{74}$ It has been studied recently for its efficacy and safety in patients with UC. Sandborn et $\mathrm{al}^{74}$ conducted an 8-week induction trial to assess the role of RPC1063 in 197 patients with moderate-to-severe disease at HD $(1 \mathrm{mg})$ and LD $(0.5 \mathrm{mg})$. They reported that HD RPC1063 was significantly superior to placebo in achieving clinical remission ( 16.3 vs $6.2, P=0.0482$ ), clinical response (58.2\% vs $36.9 \%, P=0.01)$, mucosal improvement $(34.3 \%$ vs $12.3 \%, P=0.002)$, and improvement of Mayo score from baseline (3.3 vs 1.9, $P=0.0035$ ). LD RPC1063 was inferior to $\mathrm{HD}$ and superior to placebo, but results were not statistically significant. They also found favorable safety and tolerability results for RPC1063 in the study. AEs were comparable in the groups, and the most commonly observed events were worsening of the disease, anemia, and transient elevation in ALT enzyme. ${ }^{74}$

\section{Anti-MAdCAM antibody}

MAdCAM is an adhesion molecule which is present mainly on small veins of intestines and is involved in white blood cell (WBC) trafficking from capillaries to the gut mucosa while playing its role in UC. All agents previously described have acted on integrins found on WBCs. A new method has been studied in the management of UC by inhibiting the transport of WBCs to the mucosa of intestines. Anti-MAdCAM antibodies (PF) are monoclonal antibodies against MAdCAM adhesion molecules and inhibit WBC trafficking by binding to them. ${ }^{75}$

Reinisch et al ${ }^{75}$ conducted a trial to assess the safety, efficacy, and optimal dose of PF in 357 patients with moderateto-severe UC based on Mayo score and endoscopic subscore. 
Patients were prohibited from taking other medications such as anti-TNFs, IS, and steroids before treatment with PF. They divided the patients into five groups and treated them with different doses $(7.5,22.5,75$, and $225 \mathrm{mg}$, and placebo) of PF. The end points were remission and response along with mucosal healing assessed by Mayo score and endoscopy subscore at week 12 . They reported that significantly higher percentages of patients achieved clinical remission and had mucosal healing and response in PF-treated groups rather than placebo. Furthermore, greater efficacy was observed in those patients who never had anti-TNFs as compared to the patients treated with anti-TNFs. Study also supported a favorable safety and tolerability profile of PF. Most of the observed side effects in the study were related to underlying disease with no dose-related AE reported.

\section{Colectomy}

Medical therapy is first line as well as intervention of choice in treatment of IBD. However, surgical management is an important component of therapy, since it alleviates symptoms, addresses serious complications, improves quality of life, and in some settings is lifesaving. ${ }^{76,77}$ Most common indications of colectomy in patients with SD-UC are failure of medical therapy, intractable side effects, and malignancy. ${ }^{78}$ Overall, decreasing trend of colectomy has been observed over past decades. Frolkis et $\mathrm{al}^{79}$ performed a systemic review of 486 citations from different search engines and reported the risk of surgery over 1,5 , and 10 years after the diagnosis of UC over the past 6 decades. They found significant decreasing trend in colectomy rate from $4.9 \%$ and $11.6 \%$ to $2.7 \%$ and $7.6 \%$ at 1 and 5 years, respectively, over the past 6 decades $(P<0.05)$. Surgical management is also associated with different complications such as patients may experience up to six episodes of bowel in a day and one at night as well as a risk of incontinence with increasing age. ${ }^{80}$ The most frequent AE seen following ileal pouch-anal anastomosis, is pouchitis and can be chronic in up to $19 \%$ of patients. ${ }^{80,81}$ Other AEs are intestinal obstruction, bleeding from pouch, pelvic and wound abscess, infertility, sexual dysfunction, and dysplasia and adenocarcinoma of the pouch or anal transition zone. ${ }^{82,83}$ Recently, Devaraj and Kaiser ${ }^{84}$ performed a comprehensive review of the studies and determined the difference between aggressive use of biologics and surgery in achieving treatment goals in UC. They reported that most of the UC patients do not require surgery, but patients with $\mathrm{SD}-\mathrm{UC}$ and with refractory disease who suffer from long-term immunosuppression ultimately require surgery at some stage of their life, and aggressive medical therapy in these patients with high financial burden only provides them short-term benefits and delays the need for surgery only temporarily.

\section{Optimal selection of therapy and when to switch}

As outlined earlier, there is a plethora of data on different medications used in the management of SD-UC. Recently, Bressler et $\mathrm{al}^{25}$ performed an extensive review of the literature and reached a consensus regarding therapeutic options in the management of SD-UC. The treatment algorithm can follow one of three pathways each of which is a feasible option.

1. Treatment with TPs and symptomatic response should be assessed after 8-14 weeks. Therapy should be continued with TPs in case of response, while in case of no response, TP metabolites should be measured and based upon results, dose escalation or switching of therapy should be considered.

2. Patients should be started with anti-TNFs with or without TPs/MTX, and symptomatic response should be assessed in 8-14 weeks. Therapy should be continued in case of response, while dose escalation with therapeutic drug monitoring or switching to another TNF or vedolizumab should be considered in case of no response.

3. Patients in group $\mathrm{C}$ should be started with vedolizumab with or without TPs/MTX, and symptomatic response should be assessed at 8-14 weeks. Therapy should be continued in case of response, or the treatment should be switched to anti-TNFs in case of no response.

In all the three groups, complete remission should be assessed after taking appropriate steps at 8-14 weeks, and further therapy should be tailored based upon the results. This is in accordance with the protocol of treating to target.

\section{Conclusion}

Optimal management of moderate-to-severe SD-UC is a clinical challenge. The aim of this study was to provide a detailed review of all the available options till date which have been studied in the management of SD-UC and their efficacy in the induction and maintenance of remission of the disease as well as discontinuation of CS. In the last 5 years, two anti-TNF agents and an integrin inhibitor have been approved for the management of SD-UC, and more agents are undergoing trials at the current time. The importance of treating to target and achieving steroid-free mucosal healing is being increasingly recognized, and with more aggressive therapy and the advent of newer agents, the rates of colectomy and SD disease should decrease over time. 


\section{Disclosure}

Nabeel Khan has served on speaker panels of Janssen, Abbvie, and Prometheus Laboratories Inc.; has been a member of an advisory board of GI Health Pharma; and has received grant support from Takeda and Luitpold Pharma. The other authors report no conflicts of interest in this work.

\section{References}

1. Kornbluth A, Sachar DB. Practice Parameters Committee of the American College of Gastroenterology. Am J Gastroenterol. 2010; 105(3):501-523.

2. Danese S, Sans M, Fiocchi C. Inflammatory bowel disease: the role of environmental factors. Autoimmun Rev. 2004;3(5):394-400.

3. Podolsky D. Inflammatory bowel disease. N Engl J Med. 1991;325(13): 928-937.

4. Burisch J, Munkholm P. Inflammatory bowel disease epidemiology. Curr Opin Gastroenterol. 2013;29(4):357-362.

5. Molodecky N, Soon IS, Rabi DM, et al. Increasing incidence and prevalence of the inflammatory bowel diseases with time, based on systematic review. Gastroenterology. 2012;142(1):46-54.

6. Loftus EV Jr. Clinical epidemiology of inflammatory bowel disease: incidence, prevalence, and environmental influences. Gastroenterology. 2004;126:1504-1517.

7. Kappelman MD, Rifas-Shiman SL, Kleinman K, et al. The prevalence and geographic distribution of Crohn's disease and ulcerative colitis in the United States. Clin Gastroenterol Hepatol. 2007;5: 1424-1429.

8. Truelove SC, Witts LJ. Cortisone in ulcerative colitis; final report on a therapeutic trial. Br Med J. 1955;2:1041-1048.

9. Jakobovits S, Travis SPL. Management of acute severe ulcerative colitis. Br Med Bull. 2006;75:131-144.

10. Uma Mahadevan MD. Medical treatment of ulcerative colitis. Clin Colon Rectal Surg. 2004;17(1):7-19.

11. Faubion WA Jr, Loftus EV Jr, Harmsen WS, Zinsmeister AR, Sandborn WJ. The natural history of corticosteroid therapy for inflammatory bowel disease: a population-based study. Gastroenterology. 2001;121(2):255-608.

12. Stange EF, Travis SP, Vermeire S, et al. European evidence-based Consensus on the diagnosis and management of ulcerative colitis: definitions and diagnosis. J Crohns Colitis. 2008;2:1-23.

13. Bianchi Porro G, Cassinotti A, Ferrara E, et al. Review article: the management of steroid dependency in ulcerative colitis. Aliment Pharmacol Ther. 2007;26(6):779-794.

14. Ho GT, Chiam P, Drummond H, Loane J, Arnott ID, Satsangi J. The efficacy of corticosteroid therapy in inflammatory bowel disease: analysis of a 5-year UK inception cohort. Aliment Pharmacol Ther. 2006;24(2):319-330.

15. Khan N, Abbas A, Williamson A, Balart L. Prevalence of corticosteroids use and disease course after initial steroid exposure in ulcerative colitis. Dig Dis Sci. 2013;58(10):2963-2969.

16. Khan NH, Almukhtar RM, Cole EB, Abbas AM. Early corticosteroids requirement after the diagnosis of ulcerative colitis diagnosis can predict a more severe long-term course of the disease - a nationwide study of 1035 patients. Aliment Pharmacol Ther. 2014;40(4):374-381.

17. Fraser AG, Orchard TR, Jewell DP. The efficacy of azathioprine for the treatment of inflammatory bowel disease: a 30 year review. Gut. 2002;50(4):485-489.

18. Louis E, Irving P, Beaugerie L. Use of azathioprine in IBD: modern aspects of an old drug. Gut. 2014;63(11):1695-1659.

19. Kirk AP, Lennard-Jones JE. Controlled trial of azathioprine in chronic ulcerative colitis. Br Med J (Clin Res Ed). 1982;284(6325):1 291-1292.
20. Adler DJ, Korelitz BI. The therapeutic efficacy of 6-mercaptopurine in refractory ulcerative colitis. Am J Gastroenterol. 1990;85(6): 717-722.

21. Sood A, Midha V, Sood N, Kaushal V. Role of azathioprine in severe ulcerative colitis: one-year, placebo-controlled, randomized trial. Indian J Gastroenterol. 2000;19(1):14-16.

22. Maté-Jiménez J, Hermida C, Cantero-perona J, Moreno-Otero. 6-Mercaptopurine or methotrexate added to prednisone induces and maintains remission in steroid-dependent inflammatory bowel disease. Eur J Gastroenterol Hepatol. 2000;12(11):1227-1233.

23. Ardizzone S, Maconi G, Russo A, Imbessi V, Colombo V, Bianchi Porro G. Randomised controlled trial of azathioprine and 5 -aminosalicylic acid for treatment of steroid dependent ulcerative colitis. Gut. 2006;55(1):47-53.

24. Khan KJ, Dubinsky MC, Ford AC, Ullman TA, Talley NJ, Moayyedi P. Efficacy of immunosuppressive therapy for inflammatory bowel disease: a systematic review and meta-analysis. Am J Gastroenterol. 2011;106(4):630-642.

25. Bressler B, Marshall JK, Berstein CN, et al. Clinical practice guidelines for the medical management of nonhospitalized ulcerative colitis: the Toronto consensus. Gastroenterology. 2015;148(5):1035-1058.

26. Panaccione R, Ghosh S, Middleton S, et al. Combination therapy with infliximab and azathioprine is superior to monotherapy with either agent in ulcerative colitis. Gastroenterology. 2014;146:392-400.

27. Genestier L, Paillot R, Fournel S, et al. Immunosuppressive properties of methotrexate: apoptosis and clonal deletion of activated peripheral T cells. J Clin Invest. 1998;102:322-328.

28. Genestier L, Paillot R, Quemeneur L, et al. Mechanisms of action of methotrexate. Immunopharmacology. 2000;47:247-257.

29. Rampton DS. Methotrexate in Crohn's disease. Gut. 2001;48:790-791.

30. Kozarek RA, Patterson DJ, Gelfand MD, Botoman VA, Ball TJ, Wilske KR. Methotrexate induces clinical and histologic remission in patients with refractory inflammatory bowel disease. Ann Intern Med. 1989;110(5):353-356.

31. Oren R, Arber N, Odes S, et al. Methotrexate in chronic active ulcerative colitis: a double-blind, randomized, Israeli multicenter trial. Gastroenterology. 1996;110(5):1416-1421.

32. Paoluzi OA, Pica R, Marcheggiano A, et al. Azathioprine or methotrexate in the treatment of patients with steroid-dependent or steroidresistant ulcerative colitis: results of an open-label study on efficacy and tolerability in inducing and maintaining remission. Aliment Pharmacol Ther. 2002;16(10):1751-1759.

33. Cummings JR, Herrlinger KR, Travis SP, Gorard DA, Mclntyre AS, Jewell DP. Oral methotrexate in ulcerative colitis. Aliment Pharmacol Ther. 2005;21(4):385-389.

34. Wahed M, Louis-Auguste JR, Baxter LM, et al. Efficacy of methotrexate in Crohn's disease and ulcerative colitis patients unresponsive or intolerant to azathioprine /mercaptopurine. Aliment Pharmacol Ther. 2009;30(6):614-620.

35. Mañosa M, García V, Castro L, et al. Methotrexate in ulcerative colitis: a Spanish multicentric study on clinical use and efficacy. J Crohns Colitis. 2011;5(5):397-401.

36. Baron TH, Truss CD, Elson CO. Low-dose oral methotrexate in refractory inflammatory bowel disease. Dig Dis Sci. 1993;38(10):1851-1856.

37. Khan N, Abbas AM, Moehlen M, Balart L. Methotrexate in ulcerative colitis: a nationwide retrospective cohort from the Veterans Affairs Health Care System. Inflamm Bowel Dis. 2013;19(7): 1379-1383.

38. Chande N, Wang Y, McDonald JK, McDonald JW. Methotrexate for induction of remission in ulcerative colitis. Cochrane Database Syst Rev. 2014;8:CD006618.

39. Carbonnel F. Methotrexate for corticosteroid-dependent ulcerative colitis: results of a placebo randomized controlled trial. Gastroenterology. 2015:148(4);745.

40. Owczarek D, Cibor D, Szczepanek M, et al. Biological therapy of inflammatory bowel disease. Pol Arch Med Wewn. 2009;119:84-88. 
41. Rutgeerts P, Assche G, Vermeire S. Review article: infliximab therapy for inflammatory bowel disease - seven years on. Aliment Pharmacol Ther. 2006;23(4):451-463.

42. Rutgeerts P, Sandborn WJ, Feagan BG, et al. Infliximab for induction and maintenance therapy for ulcerative colitis. N Engl J Med. 2005;353: 2462-2476.

43. Järnerot G, Ertervig E, Friis-Liby I, et al. Infliximab as rescue therapy in severe to moderately severe ulcerative colitis: a randomized, placebocontrolled study. Gastroenterology. 2005;128:1805-1811.

44. Ochsenkühn T, Sackmann M, Göke B. Infliximab for acute, not steroid-refractory ulcerative colitis: a randomized pilot study. Eur $J$ Gastroenterol Hepatol. 2004;16:1167-1171.

45. Probert CSJ, Hearing SD, Schreiber S, et al. Infliximab in moderately severe glucocorticoid resistant ulcerative colitis: a randomised controlled trial. Gut. 2003;52:998-1002.

46. Sands BE, Tremaine WJ, Sandborn WJ, et al. Infliximab in the treatment of severe, steroid-refractory ulcerative colitis: a pilot study. Inflamm Bowel Dis. 2001;7:83-88.

47. Actis GC, Bruno M, Pinna-Pintor M, et al. Infliximab for treatment of steroid-refractory ulcerative colitis. Dig Liver Dis. 2002;34:631-634.

48. Kohn A, Prantera C, Pera A, et al. Anti-tumour necrosis factor alpha (infliximab) in the treatment of severe ulcerative colitis: result of an open study on 13 patients. Dig Liver Dis. 2002;34(9):626-630.

49. Kaser A, Mairinger T, Vogel W, et al. Infliximab in severe steroid-refractory ulcerative colitis: a pilot study. Wien Klin Wochenschr. 2001;113:930-933.

50. Bermejo F, López-Sanroman A, Hinojosa J, et al. Infliximab induces clinical, endoscopic and histological responses in refractory ulcerative colitis. Rev Esp Enferm Dig. 2004;96(2):94-101.

51. Kountouras J, Zavos C, Chatzopoulos D. Anti-tumor necrosis factor therapy for ulcerative colitis. Gastroenterology. 2005;129(3):1138-1139.

52. Barreiro-de Acosta M, Lorenzo A, Mera J, Dominquez-Munoz JE. Mucosal healing and steroid-sparing associated with infliximab for steroiddependent ulcerative colitis. J Crohns Colitis. 2009; 3(4):271-276.

53. Willert RP, Lawrance IC. Use of infliximab in the prevention and delay of colectomy in severe steroid dependant and refractory ulcerative colitis. World J Gastroenterol. 2008;14(16):2544-2549.

54. Mocciaro F, Orlando A, Scimeca D, Cottone M. Terapia con infliximab nella rettocolite ulcerosa moderata-severa steroido-dipendente e steroido-refrattaria [Infliximab in moderate to severe steroiddependent or steroid-refractory ulcerative colitis]. Recenti Prog Med. 2007;98(11):560-564. Italian.

55. Armuzzi A, Pugliese D, Denese S, et al. Infliximab in steroid-dependent ulcerative colitis: effectiveness and predictors of clinical and endoscopic remission. Inflamm Bowel Dis. 2013;19(5):1065-1072.

56. Armuzzi A, Pugliese D, Denese S, et al. Long-term combination therapy with infliximab plus azathioprine predicts sustained steroid-free clinical benefit in steroid-dependent ulcerative colitis. Inflamm Bowel Dis. 2014;20(8):1368-1374.

57. Barreiro-de Acosta M, Lorenzo A, Dominguez-Muñoz JE. ADA in ulcerative colitis: two cases of mucosal healing and clinical response at two years. World J Gastroenterol. 2009;15(30):3814-3816.

58. Armuzzi A, Biancone L, Daperno M, et al. ADA in active ulcerative colitis: a "real-life" observational study. Dig Liver Dis. 2013;45:738-743.

59. Sandborn WJ, van Assche G, Reinisch W, et al. ADA induces and maintains clinical remission in patients with moderate-to-severe ulcerative colitis. Gastroenterology. 2012;142:257-265.

60. Oussalah A, Laclotte C, Chevaux J-B, et al. Long-term outcome of ADA therapy for ulcerative colitis with intolerance or lost response to infliximab a single-centre experience. Aliment Pharmacol Ther. 2008;28:966-972.

61. Reinisch W, Sandborn WJ, Hommes DW, et al. ADA for induction of clinical remission in moderately to severely active ulcerative colitis: results of a randomised controlled trial. Gut. 2011;60(6):780-787.

62. Sandborn WJ, Colombel JF, Hommes DW, et al. One-year maintenance outcomes among patients with moderately-to-severely active ulcerative colitis who responded to induction therapy with ADA: subgroup analyses from ULTRA 2. Aliment Pharmacol Ther. 2013;37(2):204-213.
63. Colombel JF, Sandborn WJ, Ghosh S, et al. Four year maintenance treatment with ADA in patients with moderately to severely active ulcerative colitis: data from ULTRA 1, 2, and 3. Am J Gastroenterol. 2014;109(11):1771-1780.

64. Acosta M. Efficacy of ADA treatment in steroid-dependent ulcerative colitis patients. United Eur Gastroenterol J. 2014:2(Suppl 1).

65. Tursi A, Elisei W, Picchio M, et al. Effectiveness of adalimumab for ambulatory ulcerative colitis patients after failure of infliximab treatment: a first "real-life" experience in primary gastroenterology centers in Italy. Ann Gastroenterol. 2014;27(4):369-373.

66. Taxonera C, Flores EI, Munoz F, et al. Adalimumab dose escalation is effective for managing loss of response in ulcerative colitis. Gastroenterology. 2015;148(4):S-789.

67. Feagan BG, Greenberg GR, Wild G, et al. Treatment of ulcerative colitis with a humanized antibody to the alpha4beta7 integrin. $N$ Engl J Med. 2005;352(24):2499-2507.

68. Garnock-Jones KP. Vedolizumab: a review of its use in adult patients with moderately to severely active ulcerative colitis or Crohn's disease. BioDrugs. 2015;29(1):57-67.

69. Feagan BG, Rutgeerts P, Sands BE, et al. Vedolizumab as induction and maintenance therapy for ulcerative colitis. $N$ Engl J Med. 2013; 369(8):699-710.

70. Parikh A, Fox I, Leach T, et al. Long-term clinical experience with vedolizumab in patients with inflammatory bowel disease. Inflamm Bowel Dis. 2013;19(8):1691-1699.

71. Arora Z, Shen B. Biological therapies for ulcerative colitis. Gastroenterol Rep. 2015;3(2):103-109.

72. Sandborn WJ, Feagan BG, et al. Subcutaneous golimumab induces clinical response and remission in patients with moderate-to-severeulcerative colitis. Gastroenterology. 2014;146(1):85-95.

73. Sandborn WJ, Feagan BG, Marano C, et al. Subcutaneous golimumab maintains clinical response in patients with moderate-to-severe ulcerative colitis. Gastroenterology. 2014;146(1):96-109.

74. Sandborn W, Feagan BG, Wolf DC, et al. The TOUCHSTONE study: a randomized, double-blind, placebo-controlled induction trial of an oral $\mathrm{S} 1 \mathrm{P}$ receptor modulator $(\mathrm{RPC1063)}$ in moderate to severe ulcerative colitis. Gastroenterology. 2015;148(4):S-93.

75. Reinisch W, Sandborn W, Danese S, et al. A randomized, multicenter double-blind, placebo-controlled study of the safety and efficacy of anti-MAdCAM antibody PF-00547659 (PF) in patients with moderate to severe ulcerative colitis: Gastroenterolgy. 2015; 148(4):S-1193.

76. Bernstein $\mathrm{CN}, \mathrm{Ng} \mathrm{SC}$, Lakatos PL, et al. A review of mortality and surgery in ulcerative colitis: milestones of the seriousness of the disease. Inflamm Bowel Dis. 2013;19(9):2001-2010.

77. Cima RR, Pemberton JH. Medical and surgical management of chronic ulcerative colitis. Arch Surg. 2005;140(3):300-310.

78. Cohen JL, Strong SA, Hyman NH, et al. Practice parameters for the surgical treatment of ulcerative colitis. Dis Colon Rectum. 2005; 48(11):1997-2009.

79. Frolkis AD, Dykeman J, Negron ME, et al. Risk of surgery for inflammatory bowel diseases has decreased over time: a systematic review and meta-analysis of population-based studies. Gastroenterology. 2013; 145(5):996-1006.

80. Li Y, Shen B. Evaluating pouch problems. Gastroenterol Clin North Am. 2012;41(2):355-378.

81. Wu H, Shen B. Pouchitis and pouch dysfunction. Med Clin North Am. 2010;94(1):75-92.

82. Waljee A, Waljee J, Morris M, Higgins PDR. Threefold increased risk of infertility: a meta-analysis of infertility after ileal pouch anal anastomosis in ulcerative colitis. Gut. 2006;55(11):1575-1580.

83. Oakley JR, Jagelman DG, Fazio VW, et al. Complications and quality of life after ileorectal anastomosis for ulcerative colitis. Am J Surg. 1985;149(1):23-30.

84. Devaraj B, Kaiser AM. Surgical management of ulcerative colitis in the era of biologics. Inflamm Bowel Dis. 2015;21(1):208-220. 


\section{Publish your work in this journal}

Clinical and Experimental Gastroenterology is an international, peerreviewed, open access journal, publishing all aspects of gastroenterology in the clinic and laboratory, including: Pathology, pathophysiology of gastrointestinal disease; Investigation and treatment of gastointestinal disease; Pharmacology of drugs used in the alimentary tract;
Immunology/genetics/genomics related to gastrointestinal disease. This journal is indexed on CAS. The manuscript management system is completely online and includes a very quick and fair peer-review system. Visit http://www.dovepress.com/testimonials.php to read real quotes from published authors.

Submit your manuscript here: http://www.dovepress.com/clinical-and-experimental-gastroenterology-journal 